\title{
New Production Relations Hiding in the New Economy
}

\author{
Wang Hong-Li ${ }^{1,2,3}$, a , Han Xiao-Pu ${ }^{1,2,3, b^{*}}$ \\ ${ }^{1}$ Hangzhou Normal University, Alibaba Complex Science Research Center, Hang Zhou, Zhe Jiang, China \\ ${ }^{2}$ Hangzhou Normal University, Institute of Information Economy, Alibaba Business School, Hang Zhou, Zhe Jiang, \\ China \\ ${ }^{3}$ Zhejiang Institute of Digital Economy and Global Coding, Hang Zhou, Zhe Jiang, China \\ awanghongli0822@126.com \\ b*xp@hznu.edu.cn;
}

\begin{abstract}
Production relations is one of the core concepts of economics, to all aspects of the society plays a decisive role Represented by Internet technique, the development of modern information technology, the most likely to promote a series of changes in social production organization form, which represent the future economy development trend of several elements of production relations is likely to have been hidden in these changes. Therefore, this paper, from the perspective of production relations, observes the organization of social production in the form of social division of labor in the fields widely recognized as the new economy, especially in internet-related industries. It is found that these changes have gone beyond the level that can be described only by factors of production, implying that the traditional industrial economic form driven by capital appreciation is transforming to a new form of production relations.
\end{abstract}

Keywords: Traditional industrial economy, The new economy, Production relations

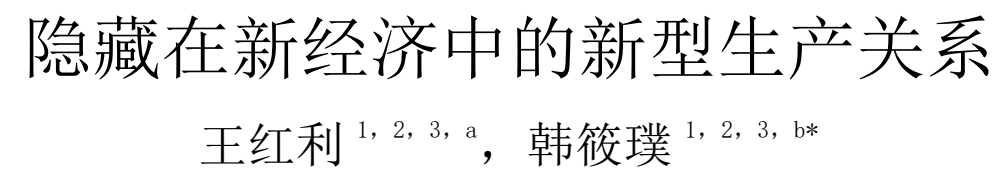

'杭州师范大学, 阿里巴巴复杂科学研究中心, 杭州, 浙江, 中国

${ }^{2}$ 杭州师范大学, 阿里巴巴商学院信息经济研究所, 杭州, 浙江, 中国

${ }^{3}$ 浙江省数字经济与全球编码研究院, 杭州, 浙江, 中国

awanghongli0822@126.com

b*xp@hznu.edu.cn;

\section{摘要}

“生产关系” 是经济学的核心概念之一，对社会的各个方面起着决定性的作用。以互联网技术为代表的现代信 息技术发展, 极有可能推动社会生产组织形态发生一系列变化, 而代表未来经济形态发展趋势的若干生产关系 元素很可能已经隐藏于这些变化之中。因此, 本文从生产关系角度入手, 观察在那些被广泛认知为 “新经济” 的领域中，特别是互联网相关产业中，在社会分工形态、社会生产的组织形态、所有制形态等生产关系层面所 发生的显性或隐性的变化, 发现这些变化已经超出了仅用生产要素所能描述的层面, 暗示着以资本增值驱动的 传统工业经济形态正在向一种全新的生产关系形态转型。

关键词: 传统工业经济; 新经济; 生产关系

\section{1. 前言}

“生产关系”是经济学的核心概念之一，它是指 经济活动中形成的所有社会关系的总称, 也是经济形 态和社会形态最直观的表现形式, 在社会的各个方面 
（包括结构、制度、文化）中起着决定性的作用 ${ }^{[1]}$ 。 通过生产关系的转变可以预测经济未来发展趋势以 及社会形态的重大转型方向。生产关系主要包含劳动 占有关系 (包括生产资料所有制、社会产品的分配关 系等) 与劳动交往关系 (包括社会分工形式、社会生 产的组织形态、商品交易形式等) 两大类型, 其中生 产资料所有制、社会分工形式与组织形态、社会产品 的分配关系等类型对生产关系的整体形态有着支配 性作用 ${ }^{[2]}$ 。

生产关系受到社会生产能力与科技水平的强烈 影响 ${ }^{[3]}$ 。传统生产关系形态是以资本驱动生产为基本 特征的传统工业经济形态。近年来, 随着互联网技术、 人工智能等现代信息技术的迅速发展, 涌现出一系列 大量的新型业态, 例如, “平台经济”、“互联网+”、

“开源社区” 等。在这一浪潮中, 经济关系形态也正 在悄然发生着变化: 一方面, 日益智能的数字平台正 在成为越来越多的经济关系的桥梁和协调中心, 产生 大量新的经济形式, 如 “共享经济”、“零工经济” 等; 另一方面, 越来越多的传统企业开始转型, “扁 平化组织”、“去中心化”、“合伙人制度”等一系 列新型组织模式和产权模式在很大程度上受到认可。 大量研究发现信息技术对这些新型业态的出现有着 重要的影响。例如, 文献 [4]认为数字技术的出现, 在组织转型和社会关系中产生了重要的作用。工作的 数字化正在创造新的工作中介方式, 例如平台在个人 之间进行在线中介, 这些所谓的在线协作平台有可能 从根本上改变劳动力市场 ${ }^{[5]}$ 。文献 [6] 提出了平台经 济, 它是基于数字平台而构建的一种经济关系, 数字 平台是通过改变我们消费和提供数字产品和服务的 方式来挑战现有企业, 利用自主代理的生态系统共同 创造价值。随着互联网的迅猛发展, 平台经济衍生出 一系列如共享经济、协同经济、零工经济等新型经济 形态。这些变化暗示着, 在信息化与智能化的驱动下, 传统的以资本增值为中心、以科层制企业为主要组织 形式的经济关系形态已经面临深刻挑战, 一种全新的 经济关系形态正在萌发。由于经济关系形态也直接驱 动着社会形态的演化, 这也预示着人类社会也将面临 一次重大转型。

\section{2. 传统工业经济的生产关系形态}

马克思在《德意志意识形态》中阐明生产、分工 以及交往是构成人类生产关系三个基本范畴 ${ }^{[2]}$ 。传统 工业经济的生产关系核心是资本驱动下的雇佣劳动 制。其主要特点包括有如下几点: 第一, 社会协作以 业缘关系为代表的熟人关系为主; 第二, 由资本所驱 动的科层制企业为社会生产的主要组织形式; 第三, 大部分劳动者不能独立展开生产, 而是受雇于企业或 各类机构, 依附于这些组织机构来实施生产并换取报 酬; 第四, 广泛采取 “专属专利” 的产权制度, 社会 资源被少数有产者控制; 第五, 获取利润, 实现资本 增值, 是企业实施生产的核心目标。之所以形成这种 形态, 社会协作对熟人关系的依赖性是其重要原因之
在这种传统工业经济的生产关系形态之下, 大多 数社会成员并没有机会直接参与核心的生产决策, 而 是依附于科层制企业。科层制企业的权力高度集中于 掌控资本的企业主, 其组织成员的地位和利益分配机 制均依赖于科层而不具有对等性，使得组织成员缺乏 自主权、决策效率低、各个部门之间协作困难等问题 ${ }^{[7]}$ 。同时, 因为把获取利润作为核心目标, 导致企业 在组织生产过程中常常出现生产与需求的偏离, 导致 负外部性 ${ }^{[8]}$ 、自然垄断 ${ }^{[9]}$ 等一系列问题。

这种生产关系形态可以适应于传统工业经济下 的市场变化缓慢、创新水平低下的稳定经济环境中。 然而, 正如下文即将展开的论述，在信息技术迅速发 展的当代，这种形态已经面临深刻挑战。

\section{3. 新经济中的新型生产关系元素}

社会协作与社会分工的发展对生产效率的提升 和社会经济的发展有着极其重要的影响。一般而言, 更高的生产效率往往依赖于社会分工的细化, 而社会 分工的细化又同社会协作水平的提高密切联系 ${ }^{[10]}$ 。在 传统经济条件下, 人们之间的协作是基于熟人为主的 业缘关系展开。当前以互联网技术为代表的现代信息 技术迅猛发展，使我们看到了社会协作突破熟人关系 的可能 ${ }^{[11]}$ 。这一突破正在推动社会生产组织形态发生 一系列变化, 呈现出向一种全新的生产关系形态演进 的趋势。这些变化或趋势可以归纳为以下几个方面:

\section{1. 陌生人之间的交易与协作愈加广泛}

以互联网为代表的信息技术发展, 使得陌生人之 间得以通过远程的信息沟通便捷地建立贸易与协作 关系。各类电子商务平台、网络金融平台, 结合了现 代物流体系, 为这些交易与协作从信息、物资、信用 等多个方面提供了支撑与保障, 推动交易成本和协作 成本的持续下降。

陌生人之间的交易与协作的广泛发展是一场革 命性的变化, 它突破了传统经济以业缘关系为代表的 熟人关系形态, 借助于互联网技术在空间关系中呈现 出一套新的远程贸易体系, 这说明一个全新的统一市 场正在基于社会网络层面形成。这一变革同工业革命 前夕由于出现农村远程贸易突破了地理位置的制约 从而建立统一大市场, 打破封建土地所有制的制约, 引发工业革命和资本主义关系的确立过程极其相似。 这暗示着, 陌生人之间的交易与协作的广泛发展不但 预示着社会分工与协作水平将发生空前提升, 而且这 个新的统一市场的形成极有可能使得社会协作全面 打破传统所有制体系下的资本限制, 最终使得资本增 值驱动机制及其各类关联形态 (如雇佣劳动制度、按 资分配制度等) 趋向消亡, 使得人类社会形态又发生 一次质的飞跃。 


\section{2. 企业中科层制组织发生改变}

当前, 以传统企业为代表的科层制组织在社会经 济运行中的角色正在趋向弱化。科层制的权力高度集 中, 具有分层管理和上下层地位不对等弊端, 使其自 身具有分工形式固化、制度僵化导致形式主义严重等 一系列缺陷, 特别是它不利于实现持续的创新 ${ }^{[12]}$ 。在 互联网时代, 市场环境快速变化, 创新水平持续提高, 大型科层制组织往往因为以上一系列的缺陷导致难 以对外界环境的变化做出快速有效的响应和调整, 难 以满足多元化的创新性市场需求 ${ }^{[13]}$ 。在这种环境下, 削弱传统科层制, 构建扁平、灵活的组织结构是企业 提高自身应变能力的常用途径 ${ }^{[14]}$ 。文献 [15]发现日益 激烈的竞争导致企业结构更加的扁平化; 文献 [16] 观察到越是靠近技术前沿的企业, 越是年轻的企业, 越有可能选择去中心化的组织结构。当前企业组织结 构的转型已经成为提升企业竞争力的重要手段 ${ }^{[17]}$ 。

目前企业组织结构转型的主要方向为开放式创 新、团队多元化、去中心化 ${ }^{[18-21]}$ 。其中开放式创新是 当前企业在创新模式方面的主要转型方向, 是指企业 可以从自己的想法和其他人的想法中创造并获利, 从 而实现创新布局的生态化，推动对市场的共同开拓 ${ }^{[22]}$ 。 而去中心化是指一种具有开放式、扁平化、平等性的 系统现象或结构, 团队多元化是指组织使用多个团队 成员来提高个人和团队的生产力和学习能力。开放式 创新和团队多元化意味着企业与企业之间的利益关 系不再有严格的壁垒, 去中心化意味着传统的科层制 开始逐步瓦解。

同时, 企业的产权体系也在逐渐发送变化, 更为 开放的合伙人制度被新兴行业企业广泛采用。随着互 联网的迅猛发展, 社会经济得到充分全面的发展, 同 时对企业要求越来越高, 而合伙人制度能够更好地满 足企业需求, 发展前景广阔。康至军认为 “知识型员 工以合伙人的身份参与工作, 不仅有利于企业的灵活 发展也有利于自身自由发展, 并且可以与企业所有者 共同分享企业利益, 从而得到激励, 提高整体创新” [23]。这一变革预示着, 合伙人制度有可能取代传统经 济中的雇佣制度。

\section{3. “个人” 正在崛起}

与传统经济形态下的雇佣关系不同, 在目前的网 络经济中, 个人成为新崛起的力量, 通过网络链接, 个体间的互动和影响日益增强, 这种凸显个人力量以 及个人之间联合的影响, 使得劳资关系由雇佣制走向 个体化发展。在一些新兴产业中涌现出越来越多的主 要依托个人影响力的独立经营者和自由职业者 ${ }^{[24]}$, 甚 至反过来影响到部分产业的业务布局, 构建起了以 “零工经济”为代表的若干类新型经济业态 ${ }^{[25]}$ 。

“零工经济” 本质上是一种短期工作形式, 指的 是用时间短、灵活的工作形式, 利用互联网和移动技 术快速匹配供需方的一种新型经济形态 ${ }^{[26]}$, 旨在促进 闲置劳动力价值再造与提升 ${ }^{[27]}$ 。在我国零工经济相关 的公司有: 滴滴出行、美团、饿了么、抖音、快手、
新浪微博、知乎等。根据 2020 年中国电子商务报告 [28]，2020 年全年，我国劳动力人口 8.8 亿，其中灵 活就业人数规模达 2 亿, 互联网灵活就业人数达 8400 万, 对缓解我国就业压力起到了重要作用。零工经济 不仅为劳动者和企业提供了双向价值, 更带来了社会 价值。这类基于 “个人” 的生产形态, 显露出对传统 经济形态中的雇佣劳动制的突破趋势。

\section{4. 资源共享理念和开放社区的出现}

随着互联网技术的快速发展, 社会资源开放共享 在部分新兴领域中已成为发展共识。“共享经济” 这 一概念最早由 Felson 和 Speath 提出 ${ }^{[29]}$, 而 Belk 将 共享现象解释为 “把属于我们自己的物品和他人一起 分享或者分享属于别人的物品的行为和过程” ${ }^{[30]}$ 。部 分学者认为, 共享经济是一种新的经济业态, 通过分 享盈余来提高资源配置的效率 ${ }^{\left[{ }^{21]}\right.}$ 。目前, 关于共享经 济这一概念业内普遍认可的定义是: 共享经济是指以 互联网和社交网络平台为基础的一种新型经济形态。 例如, 共享单车、共享充电宝、共享篮球等, 最具代 表的企业有滴滴, Uber、Airbnb 等等。

除了近年来得到广泛关注的 “共享经济” ${ }^{[29]}$, 更 为直接的资源共享机制甚至在更早的时候就已经崛 起于一些以研发性为主的开放社区中。在上世纪 90 年代伴随互联网的初期发展就已经大量出现了以 Linux 开发社区为代表的开放性社区组织 ${ }^{[32]}$, 其核心 特征是成员地位高度对等且开放共享各类资源。近年 来, 在区块链等新一代信息技术支撑下, 出现了去中 心化自治组织 (Decentralized Autonomous Organization, 缩写 DAO ) ${ }^{[33]}$ 。DAO 的含义是没有正 式的领导, 自治的意思是允许任何成员做出决定, 因 此可以把 DAO 理解成为了某一项事业把个人组织起 来, 通过创造价值来解决社会问题的自治组织。与传 统的以利润驱动为目的、公司产生的财富仅为所有者 享有并且公司所有权归小部分人享有的公司制度不 同, DAO 没有等级制度, 成员地位高度对等, 所以 DAO 所有的价值都归整体所有。同传统企业截然不同, DA0 是由任务驱动的而非利润驱动, 其主要目标是为了解 决需求或创造价值。这些开放社区的出现, 暗示着一 种由任务驱动、权力去中心化、价值共享共有、成员 对等且自由的新型生产组织形式将可能取代传统的 科层制企业成为生产组织形式的未来发展方向。

\section{5. 经济联系构建与经济决策愈发依赖信息技术}

当今的技术和社会系统是越来越大、越来越复杂 的物体。各种数据信息以指数级增长, 其增长速度已 经超过了摩尔定律 ${ }^{[34]}$ 。海量、复杂、不断增长的数据 的同时呈现，一方面使用户需要花费大量的时间进行 挑选, 另一方面大量信息容易被人忽略, 无法被大多 数用户获取。目前为止, 随着大数据时代的到来, 许 多已经成熟的以及发展中的工具都提高了我们做出 比以往更明智决定的能力。信息和通信技术对复杂大 型系统的控制和决策支持方法产生了巨大影响, 能够 帮助决策者克服在解决组织中的重要决策问题以及 
利用信息资源和分析工具帮助决策者时可能面临的 限制和制约，从而提高决策水平和质量。

推荐系统是当前典型的经济决策辅助应用。它利 用电子商务平台对各类商品与服务信息进行过滤, 根 据用户需求向用户提供相应的信息和建议, 帮助用户 从互联网数据中更全面和更精准地篮选内容。推荐系 统在购物、书籍、游戏、音乐和电影和人岗等场景均 有广泛应用 ${ }^{[35]}$ 。比如, 用于分析劳动力市场的决策支 持系统 ${ }^{[36]}$ 、用于金融部门的 IT 治理的数据驱动的决 策支持系统 ${ }^{[37]}$ 、基于兴趣度的协同过滤商品的推荐系 统 ${ }^{[38]}$ 、通过分析银行产品推荐系统设计银行产品推荐 系统模型 ${ }^{[39]}$ 、以及以患者为中心的理念, 有效提高患 者诊疗满意度的患者决策辅助系统 ${ }^{[40]}$ 。

这类依赖于机器智能的辅助决策体系是陌生人 之间建立大规模的有序协作的必需, 它实质上是一种 智能化的经济指令体系, 支撑陌生人之间可以有效地 建立经济连接。它的广泛发展, 暗示着一种指令性的 经济形态正在涌现之中。

\section{6. 创新正在成为部分产业领域的核心理念}

传统经济中, 资本增值是企业发展的基本动力。 当前, 创新所体现的价值越来越大, 与土地、机器等 生产要素的收入能力相比, 知识与智力所体现出的创 新贡献能力在收入分配中的占比在逐渐增加 ${ }^{[21]}$ 。伴随 经济活动对创新的需求越来越强烈, 创新被认为是决 定企业创造价值能力的关键因素, 已经成为企业发展 的核心决定性因素之一, 特别是在一部分知识密集型 产业领域中。这使得越来越多的经营者开始直接把 “创新” 视作企业发展的基本驱动力之一, 并直接围 绕创新来构建企业价值观。特别是对一部分知识密集 型产业领域的企业经营者而言，尽管 “盈利” 依然被 视作企业最为根本的任务, 但是创新本身正在取代直 接盈利成为推动企业发展的核心理念。企业创新过程 中所体现的协同性与开放性也在逐步增强, 多家企业 合作构建创新集群成为普遍现象。此外, 在个人化趋 势之中, 把以个人价值实现为目标的创新正在开放社 区中广泛发展 ${ }^{[41]}$ 。这一转变正在逐步削弱资本增值在 产业组织中的动力性地位, 使经济增长更为直接的面 对社会的发展性需求。

\section{4. 结论}

通过对以互联网产业为代表的一系列新兴经济 业态的分析, 我们挖掘出隐藏在互联网中的一系列新 型生产关系的萌芽, 它预示了经济发展中的趋势。特 别是, 我们观察到, 由于互联网的发展打破了地理与 空间的限制, 基于陌生人之间的协作与交易迅速增加, 并带来一系列其他生产关系层面的变化。陌生人之间 的交易迅速增加, 意味着社会成员可以直接参加市场 核心组织活动并拥有自主决策权, 不再只是依附于原 有的企业科层结构发挥自身的作用, 因此会使得企业 中科层制组织结构趋向弱化。但是随着陌生人之间的 协作的增加, 对资源共享、开放社区、外部决策机构
以及创新的要求也会随之提高, 因此伴随着信息技术 的快速发展，陌生人之间的交易迅速增加及其带来的 资源共享理念开始出现，推动了开放社区的崛起，同 时经济决策与经济联系的构建对信息技术的依赖也 越来越强。由此我们可以看出, 以上这些 “新经济” 领域在生产组织形态与社会关系构建方面的变化是 高度体系化的, 它们之间相互关联、相互促进, 其变 化已经明显超出了单纯使用生产要素所能描述的层 面, 都暗示着以资本增值为驱动力、以科层制企业为 基本生产组织单元的传统工业经济形态正在向一种 全新的生产关系形态转变, 而生产关系形态作为社会 形态的基础, 它的转变意味着社会形态的重大转型。

本文研究结论也说明, 可为陌生人之间的交易与 协作提供主要支撑的产业将在驱动经济持续发展并 推动社会转型方面扮演关键角色。它涉及到陌生人之 间的物质连接、信息连接、人员连接、价值连接等多 个方面, 有必要大力支持相关产业的发展, 并推动去 资本化运营方式在这些产业中的应用。在引导生产组 织变革方面, 去中心化的开放社区是未来组织变革的 演进方向, 暗示着有必要高度关注并大力支持如 DAO 等新型生产组织的发展与演变, 同时在理论研究层面 也需要进一步深入挖掘各类生产组织向去科层化、去 中心化方向的转型过程, 并探索对实际生产组织转型 的引导方式。

\section{项目基金}

本文为国家自然科学基金项目（62073112）的阶 段性成果之一。

\section{REFERENCES}

[1] Lange O. R. (2017) Political Economics. Wang H. C. translates, Commercial Press, Beijing.

[2] Karl M, Friedrich E. (1961) The German Ideology, People's Publishing House, Beijing.

[3] Ye S. (2016) The reform of productivity and relations of production under innovation-driven background J. Scientific Management Research, 034(005): 17-20.

[4] Nambisan S, Wright M, Feldman M. (2019) The digital transformation of innovation and entrepreneurship: Progress, challenges and key themes J. Research Policy, 48(8): 1-9.

[5] Groen W D, Maselli I. (2016) The Impact of the Collaborative Economy on the Labour Market J. Ilaria Maselli, 138: 1-35.

[6] Hein A, Schreieck M, Riasanow T, et al. (2020) Digital platform ecosystems J Electronic Markets, 30(1): 87-98.

[7] Bartlett C., Ghosha S. (1999) The Individualized Corporation. Jiangsu People's Publishing House, NanJing. 
[8] Jia M., Zhang Z., Wan D. F. (2010) Study on controlling Shareholder embezzlement and its negative externalities J. Journal of Management Science, 67:70-77.

[9] Yu L., Xiao X.Z. Summary of evolution of natural monopoly theory J. (2000) Economic Perspectives, 6:70-73

[10] Zhang F.G.,Xu Q. (2019) Adam Smith's theory of division of labor and its philosophical implication. J. Dongwu Academic,06: 104-111.

[11] Chen X, Gang L I, Jing H E. (2016) Reconstruction of chinese traditional social relationship by network society J. Journal of Beijing University of Posts and Telecommunications(Social Sciences Edition), 18(5): 8-13

[12] Gretzel, Ulrike, Yuan, et al. (2000) Preparing for the New Economy: Advertising Strategies and Change in Destination Marketing Organizations J. Journal of Travel Research, 39(2): 146-156.

[13] Yin X.J. (2020) On the reform of enterprise organization structure in Internet era J. Business Economics Research, 811(24): 109-112.

[14] Li J.X. (2014) Research on the transformation of traditional industrial production mode under the influence of Network economy[D]. Inner Mongolia Normal University.

[15] Guadalupe M, Wulf J. (2010) The flattening firm and product market competition: The effect of trade liberalization on corporate hierarchies J. American Economic Journal: Applied Economics, 2(4): 105-27.

[16] Acemoglu D, Aghion P, Lelarge C, et al. (2007) Technology, Information, and the Decentralization of the Firm J. The Quarterly Journal of Economics, 122(4): 1759-1799.

[17] Camisón C, Villar-López A. Organizational innovation as an enabler of technological innovation capabilities and firm performance [J]. Journal of Business Research, 2014, 67(1): 2891-2902.

[18] Rajan R G, Wulf J. (2006) The flattening firm: Evidence from panel data on the changing nature of corporate hierarchies J. The Review of Economics and Statistics, 88(4): 759-773.

[19] Mookherjee D. (2006) Decentralization, hierarchies, and incentives: A mechanism design perspective $\mathrm{J}$. Journal of Economic Literature, 44(2): 367-390.

[20] Huizingh E K R E. (2011) Open innovation: State of the art and future perspectives J. Technovation, 31(1): 2-9.
[21] O'leary M B, Mortensen M, Woolley A W. (2011) Multiple team membership: A theoretical model of its effects on productivity and learning for individuals and teams J. Academy of Management Review, 36(3): 461-478.

[22] Chesbrough H W. (2006) Open Innovation: The New Imperative for Creating and Profiting from Technology by Henry Chesbrough J. Academy of Management Perspectives, 20(2):86-88.

[23] Lin X., Quan G.J. (2018) A review of research on partnership system J. Modern Management Science, 11: 63-65.

[24] Acemoglu D, Aghion P, Lelarge C, et al. (2007) Technology, Information, and the Decentralization of the Firm J. The Quarterly Journal of Economics, 122(4): 1759-1799.

[25] Bloom N, Garicano L, Sadun R, et al. (2014) The distinct effects of information technology and communication technology on firm organization J. Management Science, 60(12): 2859-2885.

[26] ZhengQ., Yang W.G. (2019) A review of frontier research on gig economy J. Human Resource Development of China, 36(05): 106-115.

[27] He J., Yan S.M., Guan G. (2020) What exactly is a shared employee?-Origin, connotation, framework and trend J. Business Research, 6: 1-13.

[28] Report on E-commerce in China (2020) EB/OL http://dzsws.mofcom.gov.cn/article/ztxx/ndbg/2021 09/20210903199156.shtml

[29] Belk R. (2007) Why not share rather than own? J. The Annals of the American Academy of Political and Social Science, 611(1): 126-140

[30] Cheng M. (2016) Sharing economy: A review and agenda for future research J. International Journal of Hospitality Management, 57: 60-70.

[31] Botsman R. (2011) What's mine is yours : the rise of collaborative consumption. Harper Business UK.

[32] Du P.P., Xia H.X., Pan J.C. (2021) Research on the structural evolution characteristics of online collaborative innovation network taking Linux kernel development as an example J. Knowledge Management Forum, 2:7-13.

[33] Brafman O, Beckstrom R A. (2006) The starfish and the spider: the unstoppable power of leaderless organizations. Penguin Press, BeiJing.

[34] Chen C., Zhang C. Y. (2014)Data-intensive applications, challenges, techniques and technologies: a survey on big data J. Information Sciences, 275(1): 314-347. 
[35] Gao S., (2020) Design and implementation of cooperator recommendation System based on knowledge graph[D]. Dalian University of Technology.

[36] Brandas C, Panzaru C, Filip F G. (2016) Data Driven Decision Support Systems: An Application Case in Labour Market Analysis J. Romanian Journal of Information Science and Technology, 19(65): 65-77.

[37] Costa J. (2012) Data driven decision support systems as a critical success factor for it-governance: an application in the financial sector[D]. Lisboa: University of Lisbon.

[38] Huang G.Q., Jin F., Pengn X.Y. (2005) Collaborative filtering product recommendation system model based on interest degree $\mathrm{J}$. Microelectronics and Computers, 3: 5-8.

[39] Li X.L., Liu L. (2007) Modeling of bank product recommendation System based on collaborative Filtering J. Computer and Digital Engineering, 35(9): 6-8.

[40] He D., Zheng Y., Zhan S.Y. (2015) Development and application of patient decision aid system $\mathrm{J}$. Chinese Journal of Evidence-Based Medicine, 15(12): 1484-1488.

[41] Shao Y.Z. (2019) New economy and evolution of economic structure[D]. Henan University. 